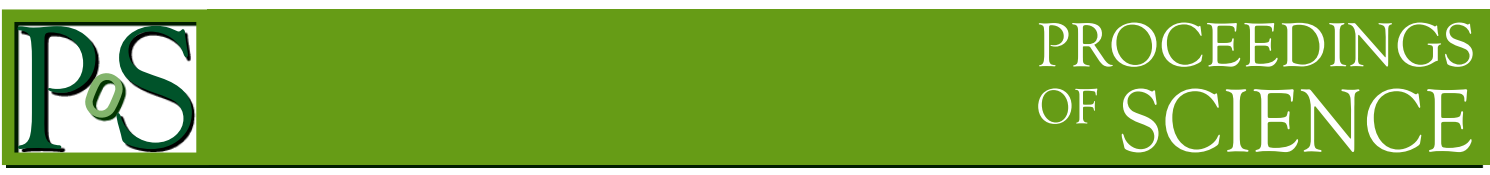

\title{
Radiation tests and production test strategy for the ALICE TOF readout upgrade board
}

\author{
Davide Falchieri ${ }^{1}$ for the ALICE Collaboration \\ INFN Bologna \\ Viale Berti Pichat 6/2, Bologna, Italy \\ E-mail: davide.falchieriabo.infn.it
}

\begin{abstract}
:
The readout board for the ALICE TOF detector named DRM2 is now in the production phase: 88 boards are being produced ( 72 are needed in the experiment). Since the board will operate in a radiation environment $(0.13 \mathrm{krad}$ total dose expected in 10 years and a flux of $0.26 \mathrm{KHz} / \mathrm{cm}^{2}$ of hadrons with energy above $20 \mathrm{MeV}$ ), a complete irradiation campaign at the component level was performed. In this paper the focus is on the irradiation tests on a Microsemi Igloo2 FPGA and two different types of Avago SFP optical transceivers using a $100 \mathrm{MeV}$ proton beam, available at the facility operated by INFN-TIFPA at the Centro di Protonterapia in Trento. This paper also focuses on the board production test strategy.
\end{abstract}

Topical Workshop on Electronics for Particle Physics (TWEPP2018)

17-21 September 2018

Antwerp, Belgium

${ }^{1}$ Speaker

(C) Copyright owned by the author(s) under the terms of the Creative Commons 


\section{Introduction}

In order to fulfill the new requirements on TOF data acquisition performance given by the higher interaction rates both in proton-proton and in lead-lead collisions during RUN3 and RUN4 at ALICE [1], an upgraded version of the readout card has been designed and is now facing the production stage. The DRM2 board [2] features a few components that are in common with the previous-generation board, like an on-board ARM-processor mezzanine (A1500) and the VME interface, and more up-to-date devices like the Microsemi Igloo2 FPGA and the GBTx ASIC [3] from CERN. An irradiation campaign was launched at the Centro di Protonterapia in Trento with the goal to quantify the board components cross section to single event upsets (SEUs) as well as to evaluate the immunity from latchup and total irradiation damages up to a dose of a few krad (factor $\sim 10-20$ related to the expected dose in the real life experiment). In a previous irradiation campaign (in 2016) several other components like static SSRAM, clock and voltage drop regulators in addition to the Igloo2 FPGA (silicon revision 1) and the optical receivers FLTL8524P28BNL and FLTL8524P28BNV from Finisar were tested. Worryingly, for the FPGA and these last two devices, single event latchups were observed.

Thus, using a $100 \mathrm{MeV}$ proton beam, we studied in 2018 three Microsemi Igloo2 FPGAs (using the latest available silicon revision number 3 ) and two sets of 4 Avago transceivers.

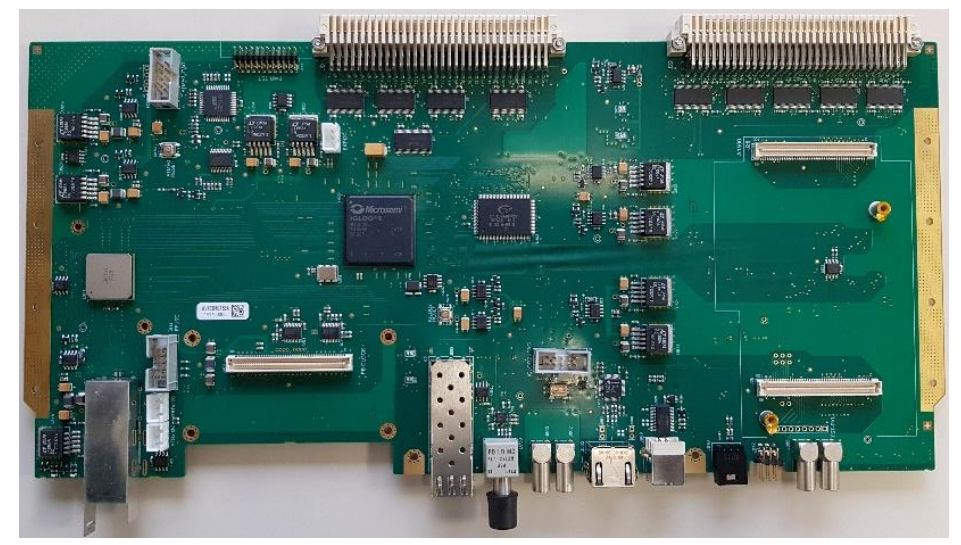

Figure 1: The DRM2 board

\section{The DRM2 board}

The DRM2 board shown in Figure 1 is the upgraded readout card for the ALICE TOF detector at CERN. Data coming from the front-end and HPTDCs are read out via VME (VME64x with 2eSST feature) and then sent towards the DAQ via the GBT link. The heart of the board is a Microsemi Igloo2 FPGA, which controls the data flow and performs all the required data formatting. An additional slow control optical link with the CONET2 protocol from CAEN is also implemented using an Igloo2 internal SERDES running at $1.25 \mathrm{Gbps}$ and a SFP optical transceiver. An on-board ARM processor on the A1500 mezzanine has the primary goal of re-programming the Igloo2 when needed: anyway, it can also be used to access Igloo2 internal memory and registers, to switch off/on the optical transceiver in case the CONET2 link gets stuck and to reset the Igloo2 FPGA. 


\section{Irradiation test setup}

For the Igloo2 irradiation test, we added some special test features to the ordinary firmware for TOF readout (see Figure 2). Two structures in particular have been inserted:

- $\quad$ an internal BRAM (64 kword of 16-bits) which is written with known patterns and then continuously read out via CONET2 and A1500. Errors in the RAM due to upsets are immediately corrected and logged.

- two 100-flip-flop D chains running at $40 \mathrm{MHz}$ which are loaded with a known pattern and then continuously checked for errors. One of the two chains is implemented with triple Modular Redundancy (TMR), the other is a simple non-protected chain.

Furthermore, the GBT link is tested in conjunction with a Xilinx KC705 evaluation board implementing the GBT FPGA core from CERN. The KC705 sends a known pattern to the Igloo2 via the GBT link: data are then sent back as in a loopback and checked against the expected values. The SFP transceivers have been irradiated while the CONET2 link was used to monitor the internal RAM to check for upsets and losses of link.

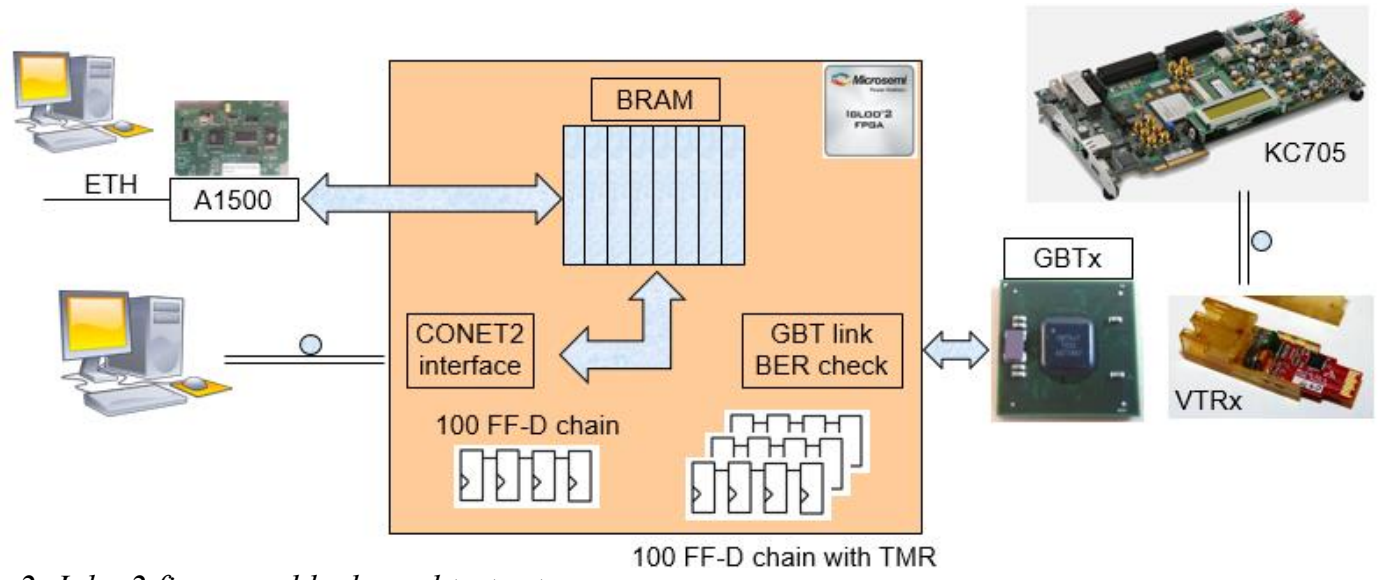

Figure 2: Igloo2 firmware blocks and test setup

\section{Irradiation results}

A total of eight Avago optical transceivers (AFBR-57R5AEZ and AFBR-57R5APZ) have been irradiated with a $100 \mathrm{MeV}$ proton beam. Table 1 reports the main results.

Table 1: Avago optical transceivers irradiation results

\begin{tabular}{|c|c|c|c|c|c|c|}
\hline Device & $\begin{array}{c}\text { Cyclotron } \\
\text { current } \\
(\mathrm{nA})\end{array}$ & $\begin{array}{c}\text { Flux } \\
(\mathrm{p} / \mathrm{s})\end{array}$ & $\begin{array}{c}\text { Duration } \\
(\mathrm{s})\end{array}$ & $\begin{array}{c}\text { Fluence } \\
(\mathrm{p})\end{array}$ & $\begin{array}{c}\text { Dose } \\
(\mathrm{krad})\end{array}$ & $\begin{array}{c}\text { Dose rate } \\
(\mathrm{rad} / \mathrm{s})\end{array}$ \\
\hline AEZ-1 & 10 & $1.0410^{8}$ & 300 & $3.1210^{10}$ & 2.93 & 9.78 \\
\hline AEZ-2 & 10 & $1.0510^{8}$ & 300 & $3.1510^{10}$ & 2.96 & 9.78 \\
\hline AEZ-3 & 10 & $1.0410^{8}$ & 450 & $4.6810^{10}$ & 4.40 & 9.78 \\
\hline AEZ-4 & 10 & $1.0410^{8}$ & 600 & $6.2410^{10}$ & 5.87 & 9.78 \\
\hline APZ-1 & 10 & $1.0410^{8}$ & 300 & $3.1210^{10}$ & 2.93 & 9.78 \\
\hline APZ-2 & 10 & $1.0410^{8}$ & 300 & $3.1210^{10}$ & 2.93 & 9.78 \\
\hline APZ-3 & 10 & $1.0410^{8}$ & 450 & $4.6810^{10}$ & 4.40 & 9.78 \\
\hline APZ-4 & 10 & $1.0410^{8}$ & 600 & $6.2410^{10}$ & 5.87 & 9.78 \\
\hline
\end{tabular}


No latchup events were observed and just one SEU event (on one AEZ type) was spotted.

Then three Igloo2 devices (M2GL090 silicon revision 3) were irradiated with the same beam. Table 2 reports the main results.

Table 2: Igloo2 FPGAs irradiation results

\begin{tabular}{|c|c|c|c|c|c|c|c|c|}
\hline Device & $\begin{array}{c}\text { Fluence } \\
(\mathrm{p})\end{array}$ & $\begin{array}{c}\text { Dose } \\
(\mathrm{krad})\end{array}$ & $\begin{array}{c}\text { BRAM } \\
\text { SEUs }\end{array}$ & $\begin{array}{c}\text { BRAM cross } \\
\text { section }\left(\mathrm{cm}^{2} / \mathrm{bit}\right)\end{array}$ & $\begin{array}{c}\text { Flip- } \\
\text { flop } \\
\text { chain } \\
\text { SEUs }\end{array}$ & $\begin{array}{c}\text { TMR } \\
\text { flip- } \\
\text { flop } \\
\text { chain } \\
\text { SEUs }\end{array}$ & $\begin{array}{c}\text { Link } \\
\text { loss }\end{array}$ & $\begin{array}{c}\text { GBT } \\
\text { link } \\
\text { SEUs }\end{array}$ \\
\hline IG1 & $1.3210^{10}$ & 1.24 & $129 \pm 11$ & $(9.32 \pm 2.5) 10^{-15}$ & 0 & 0 & 0 & 0 \\
\hline IG2 & $2.5410^{10}$ & 2.39 & $439 \pm 21$ & $(1.7 \pm 0.3) 10^{-14}$ & 0 & 0 & 0 & 0 \\
\hline IG3 & $4.0610^{10}$ & 3.82 & $605 \pm 25$ & $(2.2 \pm 0.3) 10^{-14}$ & 1 & 0 & 1 & 0 \\
\hline
\end{tabular}

No latchup events were observed and the measured BRAM cross section value for upsets $\sigma=(2.0 \pm 0.3) 10^{-14} \mathrm{~cm}^{2} / \mathrm{bit}$ is compatible with other measurements in literature [4, 5]. Using this value of cross section and the expected hadron fluence of $0.26 \mathrm{KHz} / \mathrm{cm}^{2}$, we foresee to have a SEU every 30 hours per device. Using error mitigation techniques like triple modular redundancy and error correcting code memory, we expect to further decrease the number of expected SEUs at least by a factor 2-3. The BRAM SEUs error bars are calculated with a Poisson distribution for such low level of counts.

The irradiation was performed in steps in order to check whether the FPGA was still reprogrammable after receiving a certain amount of radiation:

- IG1 and IG2 were still programmable after each irradiation step.

- IG3 was not reprogrammable immediately after the irradiation ended late in the evening. However, 12 hours later, the card was again reprogrammable, pointing to the fact a certain annealing period was needed to recover. IG3 also received a larger dose than the other devices.

Therefore, up to a few krad, re-programmability does not seem to be an issue for Igloo2 FPGAs.

\section{Production board tests}

88 DRM2 boards are currently being produced at CAEN in order to provide the 72 cards required for the experiment plus spares. The production test strategy is built upon a 3-stage approach: a first test is done at the manufacturer site (CAEN), followed by a second test performed at CERN using the same setup system as at CAEN. Before their insertion in the detector (planned during 2019), the boards are finally tested in the custom ALICE TOF VME crate where all real life functionalities (such as reading out the full crate at sustained rate) are verified together. The DRM2 has to prove to be able to acquire data from 9/10 TRM boards at the trigger rate of $33 \mathrm{kHz}$ for 2 hours. Data are then processed offline with custom tools in order to check their quality.

DRM2 boards successfully passing all the three steps will be then installed in the experimental crates, while the commissioning stage in situ will start in late 2019. 


\section{Conclusions}

The results of the irradiation test of $4+4$ Avago optical transceivers and 3 Microsemi Igloo2 FPGAs with a $100 \mathrm{MeV}$ proton beam up to a dose of a few krad were presented.

For what concerns the FPGA, irradiated with a cumulative fluence up to $410^{10} \mathrm{p} / \mathrm{cm}^{2}$, no latchup events were observed. A SEU cross section on the internal BRAM as low as $210^{-14} \mathrm{~cm}^{2}$ per bit was also measured. It is worth mentioning that all the FPGAs were still reprogrammable after receiving a dose of $3.8 \mathrm{krad}$ even if, in one case, a certain annealing time was needed before the FPGA recovered the reprogramming capability. Concerning the Avago optical transceivers, a sample of 4 AFBR-57R5AEZ and 4 AFBR-57R5APZ was used. No-latchup events and just one SEU on the former type were observed.

\section{Acknowledgements}

The author wishes to thank Francesco Tommasino, Marta Rovituso, Christian Manea and Enrico Verroi (TIFPA, Centro di Protonterapia, Trento) for the continuous support and help during the irradiation tests. The author also wishes to thank Casimiro Baldanza, Marco Giacalone, Roberto Preghenella and Pietro Antonioli (INFN Bologna) for sharing the effort of building the needed test setup and all the firmware/software required.

\section{References}

[1] K. Aamodt, The ALICE experiment at the CERN LHC, Journal of Instrumentation, volume 3, p. S08002, August 2008.

[2] D. Falchieri, P. Antonioli, C. Baldanza, F.M. Giorgi, A. Mati, C. Tintori, DRM2: the readout board for the ALICE TOF upgrade, Proceedings of Science, TWEPP-17 (2018) 081, doi: $0.22323 / 1.313 .0081$.

[3] P. Leitao et al, Test bench development for the radiation hard GBTX ASIC, Journal of Instrumentation, vol. 10, p. C01038, January 2015.

[4] Microsemi (October 2014), IGLOO2 and SmartFusion2 $65 \mathrm{~nm}$ Commercial Flash FPGAs, Interim Summary of Radiation Test Results, 51000013-2/10.14 [online]. Available at www.microsemi.com.

[5] Chengxin Zhao, A radiation tolerance study of the ALICE TPC Readout Control Unit 2, CERNTHESIS-2017-388 [online]. Available at http://cds.cern.ch. 